\title{
Robust Non-Coherent Timing Acquisition in IEEE 802.15.4a IR-UWB Networks
}

\author{
Manuel Flury*, Ruben Merz ${ }^{\dagger}$, Jean-Yves Le Boudec* \\ * EPFL, School of Computer and Communication Sciences, $\dagger$ Deutsche Telekom Laboratories, TU Berlin \\ manuel.flury@epfl.ch, ruben.merz@telekom.de, jean-yves.leboudec@epfl.ch
}

\begin{abstract}
Non-coherent energy-detection receivers are an attractive choice for IEEE 802.15.4a networks. They can exploit the ranging capabilities and the multipath resistance of impulseradio ultra-wide band (IR-UWB) at a low complexity. However, IEEE 802.15.4a receivers operate with interference created by uncontrolled piconets and an uncoordinated medium access control layer. The performance of an energy-detection IR-UWB receiver is greatly degraded in such scenarios, for both timing acquisition and decoding. In this paper, we focus on timing acquisition: we present PICNIC, a robust and low-complexity algorithm that allows for reliable timing acquisition with an IR-UWB energy-detection receiver in the presence of multiuser interference (MUI), even in near-far scenarios. At the cost of a negligible performance reduction in single-user scenarios, PICNIC outperforms classic timing acquisition algorithms by up to two orders of magnitude if MUI is present. Furthermore, PICNIC exhibits a near perfect capture property: if several transmitters compete for timing acquisition at the receiver, one signal will be acquired with practically no false detection.
\end{abstract}

\section{INTRODUCTION}

IEEE 802.15.4 targets low data-rate wireless networks with extensive battery life and very low complexity. The IEEE 802.15.4a amendment [1] specifies an impulse-radio ultrawide band (IR-UWB) physical layer for 802.15.4 networks.

Because of low complexity and low sampling requirements, energy-detection receivers are appealing to exploit the ranging capabilities and multipath resistance of IR-UWB. Compared to a coherent Rake receiver, they operate at a lower sampling frequency, are robust to timing impairments, and do not need to perform a full channel estimation.

However, IEEE 802.15.4a allows for several independent networks to operate simultaneously in the same frequency band. In addition, the mandatory medium access control (MAC) scheme is uncoordinated. Hence, IEEE 802.15.4a receivers will typically operate in the presence of multi-user interference (MUI) and near-far configurations. Prior work [2] showed that the performance of IR-UWB energy-detection receivers is greatly affected by MUI. A careless design of the receiver can cut down the benefits offered by the robustness of UWB to MUI. Furthermore, both data decoding and timing acquisition are affected.

One partial solution to prevent MUI is to coordinate access to the physical layer. However, this cannot prevent MUI due

The work presented in this paper was supported (in part) by the National Competence Center in Research on Mobile Information and Communication Systems (NCCR-MICS), a center supported by the Swiss National Science Foundation under grant number 5005-67322 to uncontrolled activities in neighboring networks. Another possibility is to revert to a coherent architecture with a Rake receiver. Such a receiver would be more robust against MUI, but at the cost of dramatically increased receiver complexity.

Instead, our approach is to design the receiver by taking MUI into account. We can then combine both the low complexity features of energy-detection receivers and robustness to MUI. MUI in low data-rate IR-UWB networks is nonGaussian [3], and its probability density function exhibits an impulsive shape [4]. With non-Gaussian interference, [5] suggests applying a non-linear function on the received signal prior to demodulation, for instance, a simple thresholding operation. Further, [6] shows the benefits of a thresholding structure on the achievable rate in IR-UWB networks. Thresholding can also be applied for timing acquisition, see e.g. [7].

Our contribution is a robust timing acquisition algorithm for IR-UWB energy-detection receivers. Our algorithm comprises an adaptive thresholding structure and an interference mitigation technique taking advantage of the knowledge of all the preamble codes in a given frequency band (see Section II). It combines robustness to MUI with the low-complexity of energy-detection. Further, it is compliant with IEEE 802.15.4a.

Thresholding was already used in [7], but not for energydetection receivers. Furthermore, the thresholds in [7] are not adaptive and must be set prior to operating the receiver. The work in [8] is also of interest since it addresses energydetection and ranging. It uses non-linear filtering that is not adaptive. The work in [9] assumes a coherent receiver and the performance in strong near-far scenarios is not evaluated. Further, issues specific to IEEE 802.15.4a are not addressed.

In the remainder of this paper we discuss the system model and assumptions in Section II. We describe our robust algorithm in Section III, and evaluate its performance in Section IV. Section V concludes the paper.

\section{System Model And Assumptions}

We consider an IEEE 802.15.4a IR-UWB physical layer [1]. Modulation is binary pulse position (BPPM) and reception is non-coherent with energy-detection. An IEEE 802.15.4a packet consists of a preamble followed by a payload. The preamble is known to the receiver and used for packet detection, timing acquisition and channel estimation. The payload carries the actual information bits to be transmitted. The preamble comprises two parts: the SYNC part used for timing acquisition and channel estimation, and the start frame 


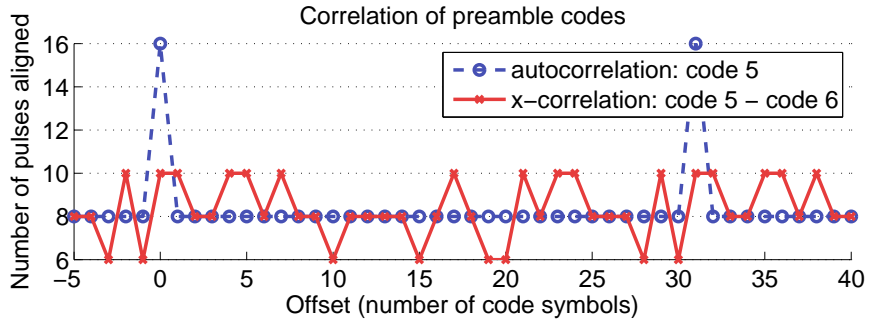

Fig. 1. Auto-correlation and cross-correlation of the two IEEE 802.15.4a preamble codes 5 and 6 of length $C=31$ when non-coherent reception is used. The cross-correlation shows 10 peaks per period that may cause false alarms.

delimiter (SFD). The SFD marks the beginning of the payload. Since robust channel estimation and SFD detection are not addressed in this paper, we detail only the SYNC part.

The time unit is a chip of duration $T_{c}$. During the SYNC part, pulses are sent at regular time intervals, every $L$-th chip. The preamble pulses are modulated according to a ternary preamble code of length $C$ without time-hopping. The SYNC part is formed by $N_{\text {sync }}$ repetitions of the preamble code.

Our receiver model comprises an antenna, a bandpass filter, a squaring device, and an integrator sampled at rate $1 / T$. Prior to integration and sampling, a received SYNC preamble signal is given by $r(t)=x\left(t-\tau_{0}\right)+w(t)$ where $x(t)$ is the contribution of the user of interest (UOI), $\tau_{0}$ is the propagation delay and $w(t)$ accounts for MUI and thermal noise. MUI is created by interfering IEEE 802.15.4a devices using the same physical layer as the UOI. Thermal noise is a zero-mean AWGN process with power spectral density (PSD) $N_{0} / 2$. We assume the noise PSD is known. It is generated by the receiver circuitry and can be calibrated or estimated in a robust fashion (see e.g. [10]). The signal of the UOI is given by

$$
x(t)=\sum_{i=0}^{N_{\mathrm{sync}}-1} \sum_{j=0}^{C-1} s_{j} \cdot h\left(t-(j+i C) L T_{c}-\tau_{0}\right)
$$

where $s_{j} \in\{-1,0,+1\}$ is the preamble code used by the UOI and $h(t)$ denotes the unknown channel response (assumed invariant for the duration of one packet and including the transmitted waveform, the response of the multipath channel and the bandpass filter). The discrete time signal after sampling is

$$
y_{n}=\int_{n T}^{(n+1) T}[r(t)]^{2} \mathrm{~d} t .
$$

We assume that $T=\frac{L}{M} T_{c}$ and $M$ is a divisor of $L$. As $L T_{c}$ is the minimum inter-pulse spacing, we obtain $M$ discrete samples $y_{n}$ per pulse.

In contrast to the preamble signal, each symbol of an IEEE 802.15.4a payload is composed of a short, continuous burst of $L_{b}$ pulses with pseudo-random polarity and time-hopping. Hence, a received payload signal stemming from an interfering transmitter contains roughly $L_{b}$ times more energy than an interfering preamble pulse.

a) Preamble Code Assignment in IEEE 802.15.4a: There are two possible preamble codes allocated for each frequency band. While they have perfect autocorrelation, the same is not true for their cross-correlation [2] (see also Figure 1). b) Statistic of the Output of the Receiver: If $x(t)=0$ and $w(t)$ is purely AWGN, the distribution of $y_{n}$ can be approximated with a chi-square distribution with $2 B T$ degrees of freedom whose cumulative distribution function we denote by $F_{\chi_{2 B T}^{2}}$ from here on.

\section{Timing ACQuisition Algorithms}

We compare three timing acquisition algorithms with increasing degree of robustness to MUI. The "baseline" algorithm uses correlation with a known template. This approach is vulnerable to MUI. The "power-independent detection" (PID) enhances the baseline algorithm using thresholding (PID was developed in [7] for coherent reception). Finally, "power-independent detection and preamble code interference cancelation" (PICNIC) adds an interference cancelation (IC) scheme tailored to IEEE 802.15.4a. All three algorithms are used for coarse timing acquisition. They usually synchronize on the strongest multipath component, which is not always the first in time. Coarse timing acquisition is then followed by a fine timing acquisition to improve the timing accuracy. Due to space restrictions, we leave out discussion of fine timing acquisition. However, any fine timing acquisition can be combined with our timing acquisition schemes.

\section{A. Baseline Algorithm}

The baseline algorithm is a classic timing acquisition using a correlation of the receiver output with a template derived from the known preamble code sequence of the UOI. A template $t_{i}$ of length $M_{T}=N_{G} \cdot C \cdot M$ is formed by repeating the preamble code $N_{G}$ times to obtain processing gain. The code symbols forming the template are squared due to non-coherent reception. Hence $t_{i}=\sum_{k=0}^{N_{G}-1} \sum_{j=0}^{C-1} s_{j}^{2} \cdot \delta(i-(j+k C) M)$. The (discrete) correlation output that follows is

$$
z_{n}=\sum_{i=0}^{M_{T}-1} t_{i} \cdot y_{n-\left(M_{T}-1\right)+i}
$$

The preamble of the UOI is $L T_{c} \cdot C$-periodic. Consequently $z_{n}$ is $M \cdot C$-periodic if the UOI signal is present. Therefore, the baseline algorithm processes the correlation output by blocks of $M C$ consecutive samples. The $i$-th block is

$$
\mathbf{z}_{\mathbf{i}}=\left\{z_{i M C}, z_{i M C+1}, \ldots, z_{(i+1) M C-1}\right\}
$$

The baseline algorithm has two steps: detection and verification. During detection, the presence of a signal is declared if at least one of the correlation output samples of the current block exceeds the threshold $\nu_{\text {base }}$. Since the statistics of the receiver output (2) are known if the received signal is AWGN only (see Section II), we have

$$
\nu_{\text {base }}=\frac{N_{0}}{2} F_{\chi_{2 B T \cdot C_{1} \cdot N_{G}}^{2}}^{-1}\left(1-P_{\mathrm{AWGN}}^{\mathrm{base}}\right) .
$$

where $C_{1}=\sum_{j=0}^{C-1} s_{j}^{2}$ denotes the number of non-zero code symbols of the UOI preamble code. The product $C_{1} \cdot N_{G}$ corresponds to the number of samples that are combined due to the template. The threshold is set by fixing the design parameter $P_{\text {AWGN }}^{\text {base }}$, which is the probability that AWGN only can exceed the threshold. If the presence of a signal is detected in the $i$-th 
block, we declare initial timing acquisition on the sample with $i$-th block index $j_{i}^{\max }$, having the highest correlation output

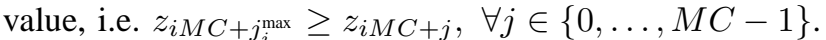

In the verification phase, we require that for every block $i+$ $k, k=1, \ldots, N$, the maximum value $z_{(i+k) M C+j_{i+k}^{\max }}>\nu_{\text {base }}$ and that its index $j_{i+k}^{\max }$ does not differ by more than the minimum inter-pulse distance $M$ from the current synchronization point $j_{i+k-1}^{\max }$ of the preceding block. This ensures that both maxima stem from the same preamble pulse. If verification is fulfilled for $N$ consecutive blocks, the verification phase succeeds and synchronization is declared. If one verification fails, synchronization starts anew with the detection phase.

The baseline method works well in a single user scenario but does not take MUI into account. A strong interfering signal has a high likelihood of exceeding the threshold (solely based on the noise level), even if not perfectly aligned with the template. This can generate missed detections (MD) if the interfering signal introduces spurious maxima in the correlation output that make the verification fail. It can lead to a false alarm (FA) with synchronization on an interfering signal if $N+1$ interfering maxima are aligned.

\section{B. Power-Independent Detection Using Thresholding}

The PID prevents large interference terms in (2) to dominate the result of the correlation given by (3) by applying a threshold check to the received signal at the input of the correlation (in contrast to the the baseline method where it is performed on the output). Samples above the threshold are set to 1 , samples below to 0 . The correlation output becomes

$$
z_{n}=\sum_{i=0}^{M_{T}-1} t_{i} \cdot \mathbf{1}_{\left[y_{n-\left(M_{T}-1\right)+i}>\nu_{\mathrm{pid}}\right]}
$$

where $\mathbf{1}_{[\cdot]}$ denotes the indicator function. The threshold $\nu_{\text {pid }}$ is given by

$$
\nu_{\text {pid }}=\frac{N_{0}}{2} F_{\chi_{2 B T}^{2}}^{-1}\left(1-P_{\mathrm{AWGN}}^{\mathrm{pid}}\right)
$$

and parameterized by $P_{\mathrm{AWGN}}^{\mathrm{pid}}$, the probability that a pure noise signal can exceed the threshold $\nu_{\text {pid }}$.

Except for the threshold check against $\nu_{\text {base }}$, the remaining steps of the baseline method are unchanged. In contrast to the original PID description in [7], where the involved thresholds were obtained through extensive simulations, our algorithm uses the explicit threshold computation of equation $(7)^{1}$.

\section{Preamble Code Interference Cancelation}

The two IEEE 802.15.4a preamble codes used per frequency band do not have a perfect cross-correlation [2]. This is shown in Figure 1 for the code sequences 5 and 6 , of length $C=31$. While code 5 has a periodic auto-correlation with only one peak per period, its cross-correlation with code 6 shows 10 peaks per period. These cross-correlation peaks can generate FAs for a receiver using code 5 . The resulting performance loss is significant (see Section IV).

\footnotetext{
${ }^{1}$ The so-called elementary threshold from [7] corresponds to $\nu_{\text {pid }}$ and the main threshold is omitted as we simply track the maximum over blocks of the correlation output.
}
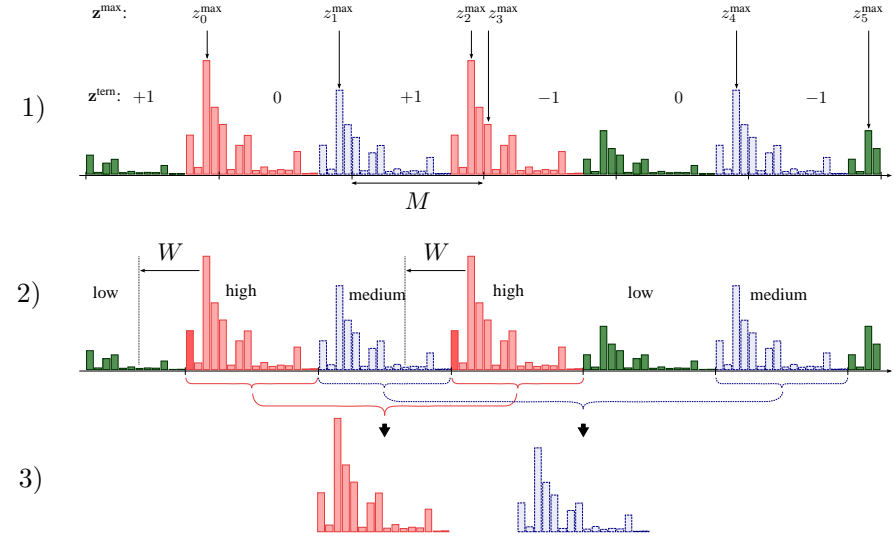

Fig. 2. Three steps of the PICNIC algorithm to cancel the effect of interfering code: 1) Interference is detected by matching cross-correlation pattern to high-, mid- and low-energy blocks in the correlation output. (2) Time-base is aligned on the interferer to find beginning of blocks via a search-back algorithm. 3) Channel energy-delay profile to be subtracted is calculated separately for high-, mid- and low-energy blocks via robust method based on order statistics.

The PICNIC algorithm attempts to detect and cancel out interference by looking for the pattern of the cross-correlation and subtracting it from the correlation output if present. PICNIC essentially pre-processes each block $\mathbf{z}_{\mathbf{i}}$, obtained from the PID correlation output in (6), before handing it over to the coarse synchronization. For convenience we omit the index $i$ from here on. If interference is present, $\mathbf{z}$ contains $C_{\text {peak }}$ sub-blocks of length $M$ with high energy, corresponding to the $C_{\text {peak }}$ peaks in the cross-correlation. It also contains $C_{\text {trough }}$ sub-blocks with low energy, corresponding to the $C_{\text {trough }}$ troughs in the cross-correlation. The remaining $C_{\text {mid }}=$ $C-C_{\text {peak }}-C_{\text {trough }}$ blocks have a medium energy level. The algorithm proceeds in three steps: 1) compare the positions of the high-, medium- and low-energy sub-blocks with the cross-correlation and decide whether interference is present 2) if present, find the exact beginning of the sub-blocks such that 3) sub-blocks with similar energy levels can be averaged yielding an estimate of the channel-energy delay profile that can be subtracted. These steps are detailed in the following and illustrated in Figure 2. In what follows the mandatory frequency band 3 with codes 5 and 6 serves as an example. However, the method equally applies to the other frequency bands with other codes. Also, as IEEE 802.15.4a allows two codes per frequency band, the knowledge of a single crosscorrelation per band is sufficient.

1) Detecting the Presence of an Interfering Preamble Code: PICNIC tries to identify sub-blocks with energy levels corresponding to the cross-correlation pattern. Two ternary vectors of length $C, \mathrm{x}^{\text {tern }}$ and $\mathbf{z}^{\text {tern }}$ are correlated, representing the different energy levels of the cross-correlation and of the $C$ sub-blocks of $\mathbf{z}$, respectively.

$\mathbf{z}^{\text {tern }}$ is constructed from $\mathbf{z}$ (see Figure 2-1 for an illustration) by first determining the maximum over every sub-block of length $M$ yielding vector $\mathbf{z}^{\max }$ with elements

$$
z_{j}^{\max }=\max \left(z_{j M}, z_{j M+1}, \ldots, z_{(j+1) M-1}\right), j \in\{0, \ldots, C-1\}
$$

Second, $\mathbf{z}^{\max }$ is converted to the ternary vector $\mathbf{z}^{\text {tern }}$ by replacing its $C_{\text {peak }}$ highest values with " +1 ", its $C_{\text {trough }}$ lowest 
values with "- 1 " and the rest with " 0 ". The cross-correlation is mapped to a ternary vector $\mathrm{x}^{\text {tern }}$ following the same procedure.

To detect interference, $\mathbf{z}^{\text {tern }}$ is correlated with $\mathrm{x}^{\text {tern }}$ and the maximum of the correlation is compared with the threshold

$$
\left\lfloor\frac{C_{\text {peak }}+C_{\text {trough }}}{2}\right\rfloor+1
$$

i.e., we test that more than half of the peaks and troughs of the cross-correlation correspond to the peaks and troughs of the sample vector. If above the threshold, we assume interference is present and continue the algorithm. Otherwise, we continue the timing acquisition according to the PID method.

2) Determination of the First Multipath Component: If interference is present, it needs to be subtracted from the vector z. Hence, a rough estimate of the channel energy-delay profile of the interfering signal must be obtained. This implies that the first multipath component of the interfering signal must be found. We use the following search-back procedure.

First, using a majority vote on the indices of the samples $z_{j}^{\max }$ of the $C_{\text {peak }}$ high energy sub-blocks corresponding to a " +1 " in $\mathbf{z}^{\text {tern }}$, the index of the strongest path into a subblock of size $M$ is determined. Second, for each of the $C_{\text {peak }}$ high-energy sub-blocks, we start from the strongest path and search in a window of length $W$ the first path above the noise threshold given by

$$
\nu_{\text {pid }}^{\mathrm{sb}}=\frac{N_{0}}{2} F_{\operatorname{Bin}\left(C_{1} N_{G}, P_{\mathrm{AWGN}}^{\mathrm{pid}}\right)}\left(1-P_{\mathrm{AWGN}}^{\mathrm{pid}, \mathrm{sb}}\right) .
$$

where $F_{\mathrm{Bin}\left(C_{1} N_{G}, P_{\mathrm{AGGN}}^{\mathrm{pid}}\right)}$ is the cumulative distribution function of the binomial distribution with parameters $C_{1} \cdot N_{G}$ and $P_{\mathrm{AWGN}}^{\mathrm{pid}}$. This corresponds to the distribution of the correlation output (6) if only AWGN is present. The threshold is set by fixing $P_{\mathrm{AWGN}}^{\text {pid, sb }}$, the probability that AWGN can exceed the threshold. Finally, the first path index is the lowest one found by more than half of the $C_{\text {peak }}$ individual search procedures.

3) Interference Cancelation by Subtraction of the Estimated Channel Energy Delay Profile: When aligned with the interfering signal, $\mathbf{z}$ is split up into $C_{\text {peak }}$ high-energy sub-blocks, $C_{\text {trough }}$ low-energy sub-blocks and $C_{\text {mid }}$ medium-energy subblocks. The signal is wrapped around if needed (see Figure 2 for the first low-energy sub-block). Then, the energy-delay profile $\mathbf{e}^{\text {high }}=\left\{e_{0}^{\text {high }}, e_{1}^{\text {high }}, \ldots, e_{M-1}^{\text {high }}\right\}$ is estimated for the high-energy sub-blocks as follows.

Let $z_{i, j}^{\text {high }}$ denote the $j$-th sample of the $i$-th high-energy subblock. We find $\mathbf{e}^{\text {high }}$ according to

$$
e_{j}^{\text {high }}=\operatorname{median}\left(z_{0, j}^{\text {high }}, z_{1, j}^{\text {high }}, \ldots, z_{C_{\text {peak }}-1, j}^{\text {high }}\right)-w_{\text {AWGN }}
$$

where the median is used instead of the mean to be robust to outliers (which might include e.g. the signal of the UOI that we do not want to subtract) and $w_{\mathrm{AWGN}}=C_{1} \cdot N_{G} \cdot P_{\mathrm{AWGN}}^{\mathrm{pid}}$ is the expected noise level at the output of the correlation (6). To cancel interference, we can now subtract $\mathbf{e}^{\text {high }}$ from all the high-energy sub-blocks in $\mathbf{z}$. We then proceed similarly for the medium- and low-energy sub-blocks.

\section{PERFormance EVAluation}

We use a packet-based IEEE 802.15.4a system simulator. We simulate one receiver and $N_{U}$ transmitters. We assume every transmitter generates packets according to a Poisson process with rate $\lambda$ : Every transmitter puts the packets in a queue. Each packet reaching the front of the queue runs through the IEEE 802.15.4a Aloha back-off procedure with back-off exponent set to its maximum value. We simulate the maximum allowed packet size with a payload of 1016 bits. A $100 \%$ utilization corresponds then to roughly $\lambda=200$ packets/s. In all simulations, we used $\lambda=100$ packets/s for all users. Two types of interfering users are simulated: Near-far interferers with a power level $10 \mathrm{~dB}$ higher than the UOI and equal power interferers with a power level equal to the UOI.

We consider the mandatory modes of IEEE 802.15.4a: Frequency band 3 with preamble codes 5 and 6 in lowpulse-repetition-frequency (LPRF) mode. Both codes have a length of $C=31$ and the cross-correlation pattern is given in Figure 1. The length of a chip is $T_{c}=2 \mathrm{~ns}$ and the spreading factor is $L=64$. The preamble length is the default length of $N_{\text {sync }}=64$ code repetitions [1]. Our receiver samples at $T=T_{c}$. Further, it uses a template with $G=10$ repetitions of the code and $N=16$ for the verification. This set of parameters, found through simulations, keeps timing acquisition fast enough such that other tasks, e.g. channel estimation, can still be performed on the preamble. We use $P_{\mathrm{AWGN}}^{\text {base }}=0.999, P_{\mathrm{AWGN}}^{\mathrm{pid}}=0.8, P_{\mathrm{AWGN}}^{\mathrm{pid}, \mathrm{sb}}=0.9999$. Extensive simulations showed that a wide range of thresholds gives similar performance, as long as $P_{\mathrm{AWGN}}^{\mathrm{pid}}$ is not set too aggressively. The propagation channel is modeled according to the 802.15.4a residential NLOS and office LOS channel models [11]. However, the results of NLOS and LOS being very similar, we only show the results for NLOS here.

Our main performance metric is the packet synchronization error rate (SER) which includes FAs and MDs. A packet is correctly acquired if the receiver synchronizes on a multipath component of the correct preamble code symbol. The signalto-noise ratio ( $\mathrm{SNR}$ ) is defined as $\mathrm{SNR}=\frac{E_{p}}{N_{0}}$ where $E_{p}$ is the received energy per pulse (after the convolution of the pulse with the impulse response of the channel). Confidence intervals shown here are at the $95 \%$ level.

Figure 3 (left) shows the performance of the different algorithms in a near-far scenario with two near interferers. The two interferers use preamble code 6 , the UOI uses code 5. The baseline method is not robust: More than $10 \%$ of the packets are lost due to interference. On the other hand, the PID method is able to reduce the impact of large interference terms generated by interfering preambles or payloads of the near interferers. The SER is improved by about one order of magnitude at high SNR. Still, even with the PID and the use of different preamble codes, FAs occur due to the imperfect cross-correlation. The PICNIC algorithm reduces this type of interference, we gain another order of magnitude. For reference, we show the single user performance of both the baseline and the PICNIC method. Single user performance of the PID method is identical to PICNIC. In this case, the PICNIC algorithm performs slightly worse than the baseline method, which is due to the fact that the threshold $\nu_{\text {pid }}$ here also removes some useful signal information.

Figure 3 (right) shows performance for two interferers with different codes, but with power levels equal to the UOI. In the 

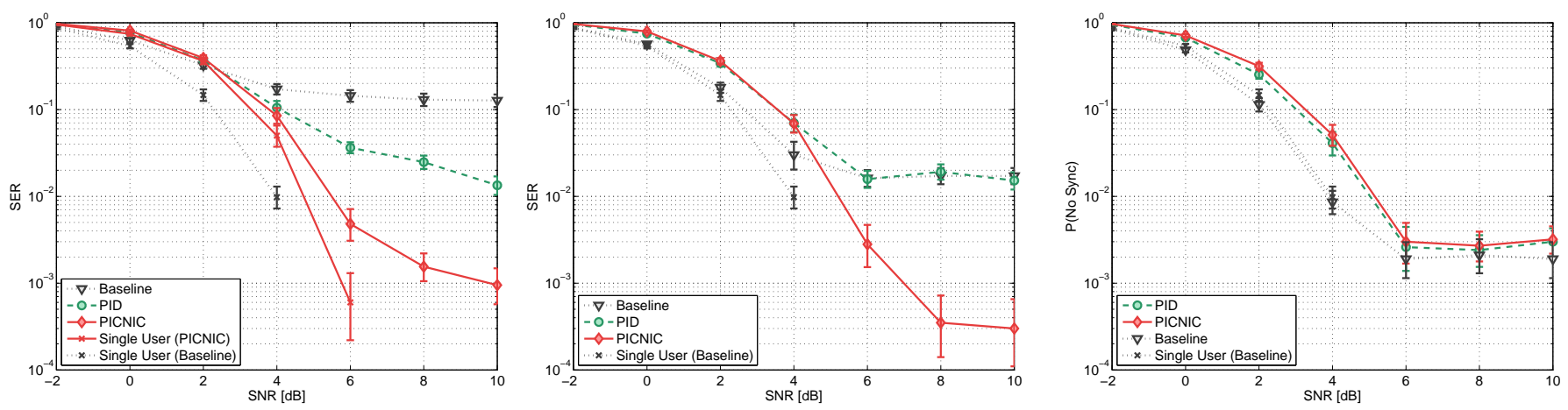

Fig. 3. SER for the different algorithms with two interferers in a near-far configuration (left) and Fig. 4. Probability of neither synchronizing to UOI with power equal to the UOI (right). Preamble codes of interferes differ from the one of the UOI. The nor an interferer for two interferers with same code baseline method is not robust. PID is able to reduce strong interference. Interference due to imperfect and same power level as the UOI. All algorithms cross-correlation is only reduced by the PICNIC method, yielding a gain of up to two orders of magnitude. show a good capture effect.

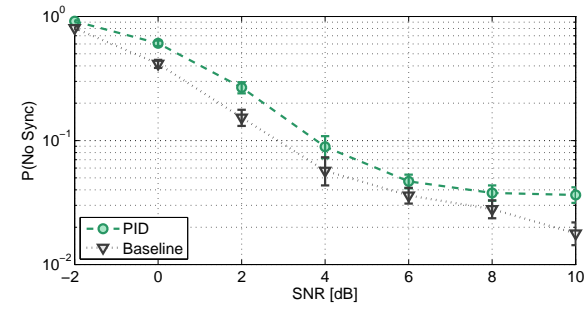

Fig. 5. Worst case scenario to assess capture effect: One equal power interferer using the same code as the UOI. Further, the interferer is always present and starts its transmission at about the same time as the UOI. Still, the probability that we acquire neither of the two is below $5 \%$.

interference limited SNR regions, the PID and the baseline methods have equal performance and show an error floor due to the imperfect cross-correlation. The PICNIC method again significantly reduces this type of interference.

If all transmitters use the same preamble codes, lots of FAs occur because the receiver cannot distinguishing an interfering signal from the signal of the UOI. Independently of the algorithms used, the SER is consequently very high (around $15 \%$ ). A more meaningful metric is needed that allows for the quantification of the capture effect capabilities of the receiver. We define the probability of no synchronization as the probability that a UOI packet is lost and the receiver does not correctly synchronize on an interfering packet either. Our simulation results show that if two packets arrive at the receiver at about the same time, the one with higher power is usually acquired. The hardest case is when these two packets have similar power levels. The verification phase may then never succeeds because the receiver switches back and forth between the two packets. To evaluate this scenario we simulate an equal-power interferer that is always present and always starts at about the same time as the UOI. The results are shown in Figure 5. For both baseline and PID (PICNIC is not shown because it coincides with PID if identical preamble codes are used), capture is above $95 \%$ at high SNR. One effect that helps here is that even though the two users have the same power level, the received energies are distributed differently because of the different propagation channels. Further, we see that baseline performs even a bit better than PID. We attribute this to the fact that the PID, to some extent, levels out different power levels through the thresholding operation on the correlation input. Figure 4 also shows the equal power scenario with identical preamble codes but here again with three users that generate packets according to a Poisson process and use the IEEE 802.15.4a Aloha back-off procedure.

\section{CONClusion}

We presented PICNIC, a low-complexity algorithm for timing acquisition with an IR-UWB energy-detection receiver in the presence of MUI. PICNIC uses a mixture of thresholding and interference cancelation and outperforms classic timing acquisition algorithms by up to two orders of magnitude if MUI is present. Furthermore, PICNIC exhibits a near perfect capture property: if several transmitters compete for timing acquisition at the receiver, one signal will be acquired with practically no false detection.

\section{REFERENCES}

[1] IEEE Computer Society, LAN/MAC Standard Committee, "IEEE P802.15.4a/D7 (amendment of IEEE std 802.15.4), part 15.4: Wireless medium access control (MAC) and physical layer (PHY) specifications for low-rate wireless personal area networks," Jan. 2007.

[2] M. Flury, R. Merz, J.-Y. le Boudec, and J. Zory, "Performance evaluation of an IEEE 802.15.4a physical layer with energy detection and multiuser interference," in IEEE ICUWB, 2007.

[3] G. Durisi and G. Romano, "On the validity of gaussian approximation to characterize the multiuser capacity of UWB TH PPM," in IEEE UWBST, 2002, pp. $157-161$.

[4] A. Forouzan, M. Nasiri-Kenari, and J. Salehi, "Performance analysis of time-hopping spread-spectrum multiple-access systems: uncoded and coded schemes," IEEE Trans. Wireless Commun., vol. 1, no. 4, 2002.

[5] A. Spaulding and D. Middleton, "Optimum reception in an impulsive interference environment-part I: Coherent detection," IEEE Trans. Commun., vol. 25, no. 9, pp. 910-923, 1977.

[6] R. Knopp and Y. Souilmi, "Achievable rates for UWB peer-to-peer networks," in IZS, 2004, pp. 82-85.

[7] A. El Fawal and J.-Y. Le Boudec, "A robust signal detection method for ultra wide band (UWB) networks with uncontrolled interference," IEEE Trans. Microw. Theory Tech., vol. 54, no. 4, pp. 1769-1781, June 2006.

[8] Z. Sahinoglu and I. Guvenc, "Multiuser interference mitigation in noncoherent uwb ranging via nonlinear filtering," EURASIP Journal on Wireless Communications and Networking, pp. 1-10, 2006.

[9] E. Ekrem, M. Koca, and H. Delic, "Robust ultra-wideband signal acquisition," Wireless Communications, IEEE Transactions on, vol. 7, no. 11, pp. 4656-4669, November 2008.

[10] M. Flury, R. Merz, and J.-Y. Le Boudec, "An Energy Detection Receiver Robust to Multi-User Interference for IEEE 802.15.4a Networks," in IEEE ICUWB, 2008.

[11] “IEEE 802.15.4a channel model - final report,” November 2004. 